\title{
Coherent combining architectures for high-brightness laser diodes
}

\author{
Gaëlle Lucas-Leclin*, Guillaume Schimmel, Philipp Albrodt, Marc Hanna, Patrick Georges \\ Laboratoire Charles Fabry, Institut d'Optique Graduate School, CNRS, Université Paris-Saclay, \\ Palaiseau, France ("gaelle.lucas-leclin@institutoptique.fr)
}

\begin{abstract}
Coherent beam combining aims at increasing the spatial brightness of lasers. It consists in maintaining a constant phase relationship between different emitters, in order to combine them constructively in one single beam. Different architectures are proposed in the literature, either based on lasers in a passive extended-cavity or amplifiers seeded by a master oscillator. We compare the different approaches for highbrightness tapered laser devices.
\end{abstract}

Index Terms-Diode lasers, Laser cavity resonators, Phase noise, Semiconductor laser arrays.

\section{INTRODUCTION}

$\mathrm{D}$ IODE lasers are the most efficient technology for converting electrical energy into useful light. However, this efficiency is not available to most industrial users due to the low brightness of edge-emitting devices. Overcoming this limitation would deliver a technological breakthrough in cost effective, high-brilliance laser diode sources for industrial applications. The favored approach to scale up the brightness is to use several moderately powerful high-brightness lasers and to combine them in one single beam [1][2]. The conservation of etendue principle forbids the improvement of spatial brightness through incoherent beam superposition. Spectral beam combining is already used in industrial laser systems, demonstrating record powers in the $\mathrm{kW}$ range with high efficiencies, but has the drawback of the inherently broad spectrum of the source that is composed of one laser line per emitter [3][4].

On the other hand, coherent beam combining (CBC) preserves all the laser properties, and is compatible with other combining techniques for further scaling of the power [5]. It consists in the superposition of individual laser beams by constructive interference, providing a single high-power laser beam. The power and spatial brightness of the combined beam increase far above what could be achieved from a single device. The major condition for the combining to be efficient is the mutual spatial and temporal coherence of the beams: their relative phases have to be constant over time with a high precision, which implies that the beams are phase-locked. The beam quality of the combined beam is improved with regard to the initial beams, as $\mathrm{CBC}$ rejects any disparity between them and acts thus as a spatial filter. Consequently the intrinsic beam quality of the individual emitters is a key parameter for the coherent combining efficiency.

In the following, we describe our most recent investigations of different $\mathrm{CBC}$ architectures, both active and passive, with high-brightness tapered lasers and amplifiers. Indeed, among semiconductor edge-emitting devices, tapered section laser diodes have demonstrated the highest power ( 5 to $8 \mathrm{~W}$ ) in a nearly diffraction-limited beam, and so the highest brightness, in the order of $1 \mathrm{GW} \cdot \mathrm{cm}^{-2} \cdot \mathrm{sr}^{-1}$, far above what is achieved from ridge emitter devices [6]. They are therefore the perfect building block for further power and brightness scaling.

\section{EXTENDED-CAVITY CONFIGURATIONS}

With laser diodes, extended-cavity configurations have aroused a particular interest as they may provide all-passive phase-locking and coherent combining. Many different configurations have been studied in the literature, that all aim at ensuring both a strong feedback of the external cavity and a significant coupling between emitters - thus favoring the phase-locked operation of the laser ensemble with regard to its incoherent emission [7][8][9]. It is noteworthy that in most cases, the extended cavity is designed to force the phaselocked operation of the laser emitters on a known phase relationship, but the coherent superposition of the laser beams, which is per se coherent combining, is realized outside the cavity with additional optical elements [10].

The easiest architecture consists in taking benefit of the rear facet mirror of the laser diodes, and having the extendedcavity on the common front facet of the laser arrays. The phase-locked power is extracted through the extended-cavity output coupler. There is thus a trade-off between strong optical feedback and high power extraction, and such architecture suffers from a low electrical-to-optical efficiency. Additionally, any optical feedback into the front section of tapered laser diodes is known to decrease their beam quality, making such approach incompatible for such devices [11].

We have investigated a novel configuration designed on the rear-side of the lasers, and based on the separation of the phase-locking and coherent combining of the beams [12][13]. It is particularly suitable for tapered laser devices, and should increase the electrical-to-optical efficiency of CBC sources. We have used an array of five individually-addressable tapered devices distant from $500 \mu \mathrm{m}$, with $\mathrm{R}<0.1 \%$ coating 
on the rear side and $\mathrm{R}=0.5 \%$ on the front facet. The extended cavity, on the rear side of the laser array, uses a diffractive optical element (DOE) as a splitter and combiner, and a highreflection (HR) mirror closes the cavity on the $0^{\text {th }}$ order of the DOE (Figure 1). Phase-locked operation of the laser array is observed up to a current of $5 \mathrm{~A}$ per device, with a high visibility up to $92 \%$ demonstrating mutual coherence among the emitters. An identical DOE is used on the front side of the laser for the coherent superposition of the phase-locked beams. The maximum combined optical power in the on-axis diffracted order reaches $7.5 \mathrm{~W}$, corresponding to a combining efficiency of $62 \%$.

\section{MOPA CONFIGURATIONS}

The Master Oscillator - Power Amplifier (MOPA) configuration is most popular for multi-kW-class fiber and solid-state amplifiers [5]. It consists in the parallel amplification of a single-frequency and low-power seed source (MO) in multiple amplifiers, followed by the coherent superposition of the amplified beams. Such an architecture is linear by essence, and simpler to implement than extendedcavity setups. Nonetheless, the phases need to be constantly corrected to maintain efficient beam combining of the amplified beams.

As the power amplifiers, we have used the same array of five tapered devices as in section II. The MO is a DFB laser diode emitting at $976 \mathrm{~nm}$, which beam is diffracted into five by the rear DOE. The relative phases of the amplified beams are automatically controlled through the current in each ridge section, with limited impact on the power extraction. The coherent superposition of the five amplified beams is realized on the front side of the array, like the previous setup [13]. The combining efficiency reaches $82 \%$ at $3 \mathrm{~A}$, and decreases to $78 \%$ at the maximum operating current $\mathrm{I}_{\mathrm{T}}=6 \mathrm{~A}$ with regard to the total power extracted from the laser bar. It is limited by the proportion of the tapered emission that is not in the combined fundamental transverse mode. The maximum combined power is $11.5 \mathrm{~W}$.

\section{CONCLUSION}

Different approaches have been proposed in order to improve the spatial brightness of edge-emitting laser diodes. Among them, phase-locking and coherent combining of an array of emitters is particularly attractive as it maintains all the laser properties (beam quality, spectrum). We have investigated and compared different $\mathrm{CBC}$ architectures dedicated to optimize the combining efficiency and power of the laser source, based on arrays of high-brightness tapered laser diodes. With only five emitters, we have achieved a combined power of $11.5 \mathrm{~W}$ in a diffraction-limited single lobe, which demonstrates the potential of tapered amplifiers for high-power and high-brightness modules based on coherent combining. The most robust architecture appears to be the MOPA one, and further increase of the output power is expected with improved tapered devices and better thermal management.

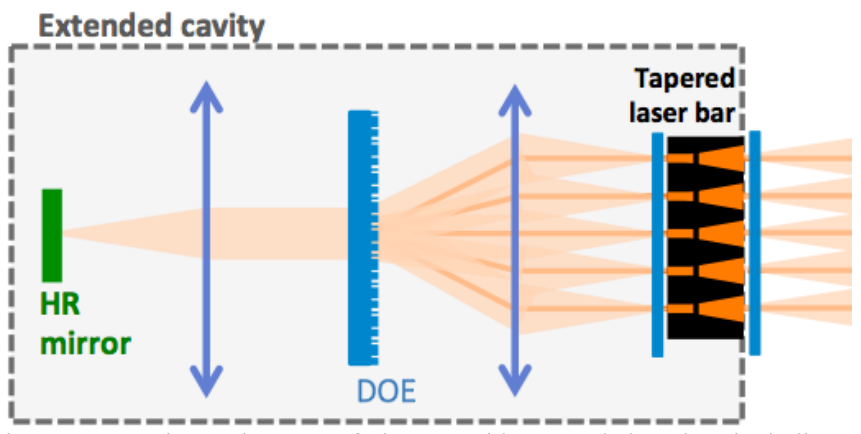

Fig. 1. Experimental setup of the rear-side extended-cavity, including a diffractive optical element (DOE), a HR mirror, two Fourier lenses and a fastaxis collimator.

\section{ACKNOWLEDGEMENTS}

This work was partly supported by the European Commission within the WWW.BRIGHTER.EU program under grant FP6-IST-035266, and the BRIDLE program under grant FP7-314719.

\section{REFERENCES}

[1] T. Fan, "Laser beam combining for high-power, high-radiance sources", IEEE J. Sel.Topics. Quantum Electron., vol. 11, no. 3, pp. 567$577,2005$.

[2] G. Schimmel, I. Doyen, S. Janicot, M. Hanna, P. Georges, G. LucasLeclin, J. Decker, P. Crump, G. Erbert, S. Kaunga-Nyirenda, D. Moss, S. Bull, E. C. Larkins, U. Witte, M. Traub, "High-power operation of coherently coupled tapered laser diodes in an external cavity," Proc. SPIE vol. 9733, 97330I, 2016

[3] R. Huang, B. Chann, J. Burgess, M. Kaiman, "Direct diode lasers with comparable beam quality to fiber, $\mathrm{CO} 2$, and solid state lasers," in Proc. SPIE 8241, 2012, 824102.

[4] U. Witte, F. Schneider, C. Holly, A. Di Meo, D. Rubel, F. Boergmann, M. Traub, D. Hoffmann, S. Drovs, T. Brand, A. Unger, "kW-class direct diode laser for sheet metal cutting based on commercial pump modules", Proc. SPIE vol. 10086, p. 1008608-1, 2017.

[5] A. Brignon, Coherent Laser Beam Combining, John Wiley \& Sons 2013.

[6] C. Fiebig, G. Blume, C. Kaspari, D. Feise, J. Fricke, M. Matalla, W. John, H. Wenzel, K. Paschke, G. Erbert, "12W high-brightness singlefrequency DBR tapered diode laser", Electronics Letters, vol. 44, no. 21, p. 1253, 2008.

[7] D. Paboeuf, G. Lucas-Leclin, P. Georges, N. Michel, M. Krakowski, J. Lim, S. Sujecki, E. Larkins, "Narrow-line coherently combined tapered laser diodes in a Talbot external cavity with a volume Bragg grating," Applied Physics Letters, vol.93, no 21, p 211102, 2008

[8] C. J. Corcoran, F. Durville, "Passive coherent combination of a diode laser array with 35 elements," Opt. Express 22(7), 8420 (2014).

[9] J. Montoya, S. J. Augst, K. Creedon, J. Kansky, T. Y. Fan, A. SanchezRubio, "External cavity beam combining of 21 semiconductor lasers using SPGD," Appl. Opt. vol 51, no 11, p1724, 2012.

[10] D. Pabœuf, F. Emaury, S. de Rossi, R. Mercier, G. Lucas-Leclin, P. Georges, "Coherent beam superposition of ten diode lasers with a Dammann grating," Opt. Lett., vol. 35, pp 1515-1517, 2010

[11] M.A. Helal, S. Bull, S.N. Kaunga-Nyirenda, J.J. Lim, E.C. Larkins, "Self-consistent simulation of high-brightness diode lasers with external optical feedback,", IEEE High Power Diode Lasers and Systems Conference, Oct. 2015, Coventry, United Kingdom.

[12] G. Schimmel, I. Doyen-Moldovan, S. Janicot, M. Hanna, J. Decker, P. Crump, G. Blume, G. Erbert, P. Georges, G. Lucas-Leclin, "Rear-side resonator architecture for the passive coherent combining of highbrightness laser diodes," Opt. Lett., vol. 41, no. 5, pp 950-953, 2016

[13] G. Schimmel, S. Janicot, M. Hanna, J. Decker, P. Crump, G. Erbert, U. Witte, M. Traub, P. Georges, G. Lucas-Leclin, "Coherent beam combining architectures for high power tapered laser arrays", Proc. SPIE vol. 10086, 100860O, 2017 